\title{
Hidden Variables in Deep Learning Digital Pathology and Their Potential to Cause Batch Effects: Prediction Model Study
}

Max Schmitt ${ }^{*}$, MSc; Roman Christoph Maron ${ }^{1 *}$, MSc; Achim Hekler ${ }^{1}$, MSc; Albrecht Stenzinger ${ }^{2}$, MD; Axel Hauschild $^{3}$, MD; Michael Weichenthal ${ }^{3}$, MD; Markus Tiemann ${ }^{4}$, MD; Dieter Krahl ${ }^{5}$, MD; Heinz Kutzner ${ }^{6}$, MD; Jochen Sven Utikal ${ }^{7,8}$, MD; Sebastian Haferkamp ${ }^{9}$, MD; Jakob Nikolas Kather ${ }^{10}$, MD; Frederick Klauschen ${ }^{11}$, MD; Eva Krieghoff-Henning ${ }^{1}, \mathrm{PhD}$; Stefan Fröhling ${ }^{12}$, MD; Christof von Kalle ${ }^{13}$, MD; Titus Josef Brinker ${ }^{1}, \mathrm{MD}$

${ }^{1}$ Digital Biomarkers for Oncology Group, National Center for Tumor Diseases, German Cancer Research Center (DKFZ), Heidelberg, Germany

${ }^{2}$ Institute of Pathology, University Hospital Heidelberg, University of Heidelberg, Heidelberg, Germany

${ }^{3}$ Department of Dermatology, University Hospital Kiel, University of Kiel, Kiel, Germany

${ }^{4}$ Institute for Hematopathology Hamburg, Hamburg, Germany

${ }^{5}$ Private Institute of Dermatopathology, Heidelberg, Germany

${ }^{6}$ Private Institute of Dermatopathology, Friedrichshafen, Germany

${ }^{7}$ Skin Cancer Unit, German Cancer Research Center (DKFZ), Heidelberg, Germany

${ }^{8}$ Department of Dermatology, University Medical Center Mannheim, University of Heidelberg, Mannheim, Germany

${ }^{9}$ Department of Dermatology, University Hospital of Regensburg, Regensburg, Germany

${ }^{10}$ Department of Medicine III, RWTH University Hospital Aachen, Aachen, Germany

${ }^{11}$ Institute of Pathology, Charité University Hospital Berlin, Berlin, Germany

${ }^{12}$ National Center for Tumor Diseases, German Cancer Research Center (DKFZ), Heidelberg, Germany

${ }^{13}$ Department of Clinical-Translational Sciences, Charité and Berlin Institute of Health, Berlin, Germany

* these authors contributed equally

\section{Corresponding Author:}

Titus Josef Brinker, MD

Digital Biomarkers for Oncology Group

National Center for Tumor Diseases

German Cancer Research Center (DKFZ)

Im Neuenheimer Feld 460

Heidelberg, 69120

Germany

Phone: 4962213219304

Email: titus.brinker@dkfz.de

\section{Abstract}

Background: An increasing number of studies within digital pathology show the potential of artificial intelligence (AI) to diagnose cancer using histological whole slide images, which requires large and diverse data sets. While diversification may result in more generalizable AI-based systems, it can also introduce hidden variables. If neural networks are able to distinguish/learn hidden variables, these variables can introduce batch effects that compromise the accuracy of classification systems.

Objective: The objective of the study was to analyze the learnability of an exemplary selection of hidden variables (patient age, slide preparation date, slide origin, and scanner type) that are commonly found in whole slide image data sets in digital pathology and could create batch effects.

Methods: We trained four separate convolutional neural networks (CNNs) to learn four variables using a data set of digitized whole slide melanoma images from five different institutes. For robustness, each CNN training and evaluation run was repeated multiple times, and a variable was only considered learnable if the lower bound of the $95 \%$ confidence interval of its mean balanced accuracy was above $50.0 \%$.

Results: A mean balanced accuracy above 50.0\% was achieved for all four tasks, even when considering the lower bound of the $95 \%$ confidence interval. Performance between tasks showed wide variation, ranging from $56.1 \%$ (slide preparation date) to $100 \%$ (slide origin). 
Conclusions: Because all of the analyzed hidden variables are learnable, they have the potential to create batch effects in dermatopathology data sets, which negatively affect AI-based classification systems. Practitioners should be aware of these and similar pitfalls when developing and evaluating such systems and address these and potentially other batch effect variables in their data sets through sufficient data set stratification.

(J Med Internet Res 2021;23(2):e23436) doi: $\underline{10.2196 / 23436}$

\section{KEYWORDS}

artificial intelligence; machine learning; deep learning; neural networks; convolutional neural networks; pathology; clinical pathology; digital pathology; pitfalls; artifacts

\section{Introduction}

The advent of artificial intelligence (AI) in digital pathology (DP) has resulted in the development of various algorithms for the detection, classification, and further evaluation of multiple cancer subtypes [1]. General interest and enthusiasm for this emerging technology continues to grow, exemplified by the development of a variety of convolutional neural network (CNN)-based oncology systems for the analysis of histological images of breast [2,3], lung [4], skin [5,6], and gastrointestinal [7] cancer. However, the successful implementation of CNN-based assistance systems in DP is complicated by a plethora of challenges [8-10], some of which are domain-specific, while others are omnipresent in the field of deep learning (DL) and machine learning (ML) in general.

One important issue in the field of biomedical data analysis is the occurrence of batch effects, which are defined as differences among subsets of a data set introduced through technological artifacts [11,12]. In DP, such artifacts are introduced during tissue processing and slide preparation [13], and presumably also during slide digitization, image compression, and storage [14], all of which affect slide and image appearance (Figure 1). We expand on this definition of batch effects by including biological factors, presumably unrelated to the actual classification task, as causative agents. Both factors (biological and nonbiological) are referred to as hidden variables from here on.

Figure 1. Comparison of exemplary whole slide image sections obtained at different institutes: (A) Institute of Pathology, University Hospital Heidelberg, University of Heidelberg, Heidelberg, Germany (Zeiss scanner; Carl Zeiss AG); (B) Department of Dermatology, University Hospital Kiel, University of Kiel, Kiel, Germany (3DHISTECH scanner; 3DHISTECH Ltd); (C) Private Institute of Dermatopathology, Mönchhofstraße 52, Heidelberg, Germany (Zeiss scanner); (D) Department of Dermatology, University Medical Center Mannheim, University of Heidelberg, Mannheim, Germany (Zeiss scanner); and (E) Private Institute of Dermatopathology, Siemensstraße 6/1, Friedrichshafen, Germany (Zeiss scanner). (F) The same slide section as shown in (E) but scanned with a Hamamatsu scanner (Hamamatsu Photonics KK) rather than a Zeiss scanner.

A

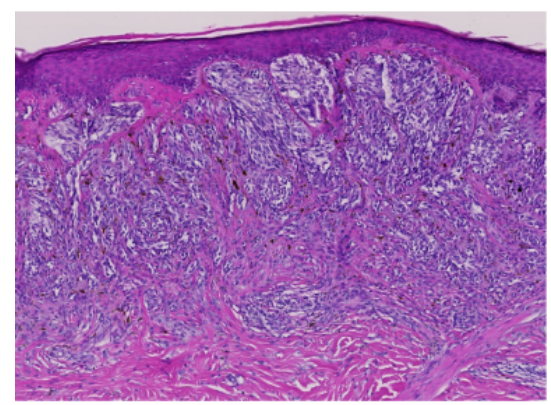

D

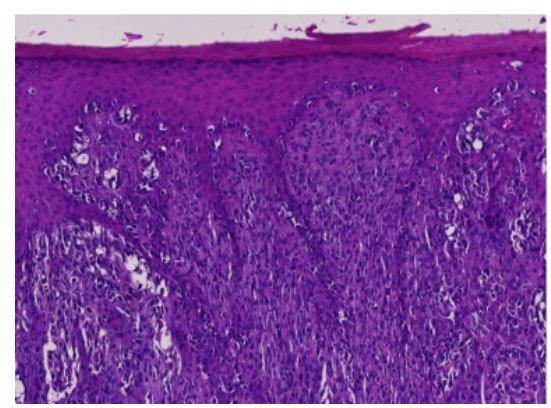

B

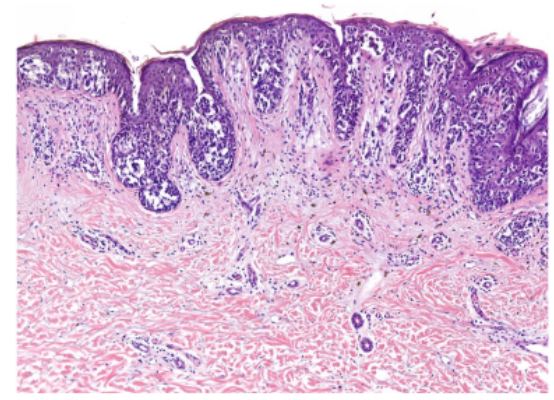

E

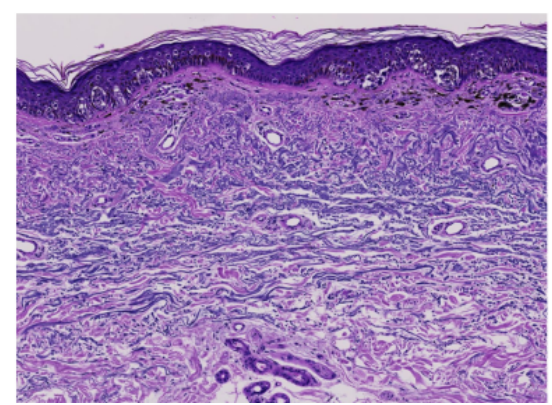

C

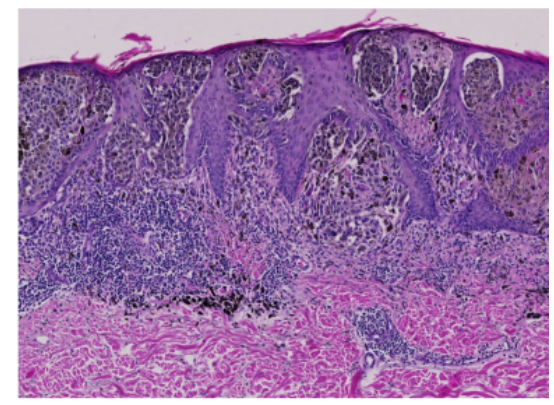

$\mathrm{F}$

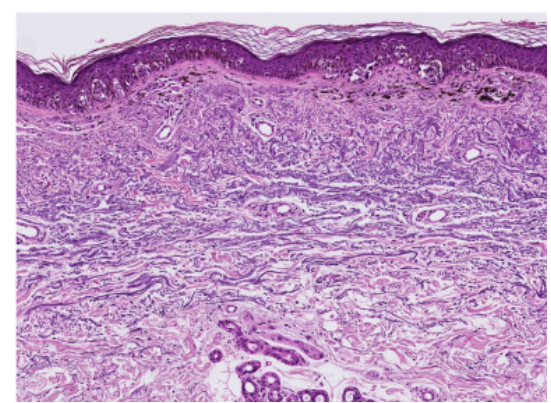

Batch effects can be problematic during development of ML models, where hidden variables are learned instead of or in addition to the intended target variables. The hidden variable then acts as a complete or partial proxy for the intended target variable, negatively affecting the model's performance. Studies have addressed this issue by focusing on various normalization techniques [15-19]. In addition, standardized preprocessing procedures and balanced data set construction may aid in reducing but not eliminating batch effects. Overall, this is concerning, and a previous study using a breast cancer tissue cohort has already suggested the existence of batch effects in 
parts of the publicly available Cancer Genome Atlas (TCGA) pathology repository [20].

We expand on these findings by analyzing the learnability of four exemplary selected hidden variables, as learnable variables can cause batch effects that can negatively influence DL algorithms. Our research on hidden variables aims to highlight that batch effects are not an unlikely occurrence, thereby reinforcing the importance of proper data set construction and experimental design, as well as sensitizing the community toward these and similar pitfalls for the emerging field of DL in DP.

\section{Methods}

\section{Study Design}

Using a proprietary dermatopathological data set of anonymized slides, a series of ML tasks were formulated, where each task investigated the learnability of a certain hidden variable. The analyzed variables are believed to be found throughout DP whole slide image (WSI) data sets, but their learnability does not necessarily need to generalize.
Next, multiple DL models of the same architecture were trained on the variable stated by each task, followed by subsequent performance analysis, where an assessment was made of whether the task's variable was learnable or unlearnable. A variable was considered learnable when the $95 \%$ confidence interval of its mean balanced accuracy had a lower bound above $50.0 \%$ when calculated on the slide level. Note that a random classifier, which is unable to learn the variable, would be expected to achieve a balanced accuracy of approximately $50.0 \%$.

Ethics approval was obtained from the ethics committee of the Medical Faculty of Mannheim, University of Heidelberg, Mannheim, Germany.

\section{Data Set}

A total of 427 hematoxylin and eosin-stained preparations were obtained from five different institutes, with each slide belonging to one patient and containing tissue sections of melanoma biopsies (Table 1). For details on the slide digitization process, see Multimedia Appendix 1.

Table 1. Overview of individual data sets.

\begin{tabular}{|c|c|c|c|c|c|}
\hline Data set & Origin of slides & Number of slides & Number of tiles & Scanner type & Tasks \\
\hline 1 & Heidelberg $^{\mathrm{a}}$ & 81 & $1,344,825$ & Zeiss $^{b}$ & $\begin{array}{l}2 \text { (slide preparation date); } 3 \text { (slide } \\
\text { origin) }\end{array}$ \\
\hline 2 & $\mathrm{Kiel}^{\mathrm{c}}$ & 196 & $2,092,726$ & 3DHISTECH ${ }^{\mathrm{d}}$ & $\begin{array}{l}1 \text { (patient age); } 2 \text { (slide preparation } \\
\text { date) }\end{array}$ \\
\hline 3 & Heidelberg ${ }^{\mathrm{e}}$ & 73 & 832,940 & Zeiss & 3 (slide origin) \\
\hline 4 & Friedrichshafen ${ }^{\mathrm{f}}$ & 54 & 350,$518 ; 364,196$ & Zeiss; Hamamatsu ${ }^{\mathrm{g}}$ & 3 (slide origin); 4 (scanner type) \\
\hline 5 & Mannheim ${ }^{\mathrm{h}}$ & 23 & 513,256 & Zeiss & 3 (slide origin) \\
\hline
\end{tabular}

${ }^{\mathrm{a}}$ Institute of Pathology, University Hospital Heidelberg, University of Heidelberg, Heidelberg, Germany.

${ }^{\mathrm{b}}$ Carl Zeiss AG.

${ }^{\mathrm{c}}$ Department of Dermatology, University Hospital Kiel, University of Kiel, Kiel, Germany.

$\mathrm{d}_{\text {3DHISTECH Ltd. }}$

${ }^{\mathrm{e}}$ Private Institute of Dermatopathology, Mönchhofstraße 52, Heidelberg, Germany.

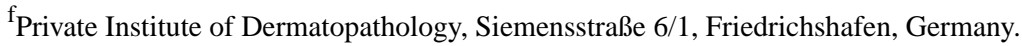

${ }^{\mathrm{g}}$ Hamamatsu Photonics KK.

${ }^{\mathrm{h}}$ Department of Dermatology, University Medical Center Mannheim, University of Heidelberg, Mannheim, Germany.

\section{Classification Tasks}

Four classification tasks were performed, each analyzing one predefined hidden variable. Data sets for each task were chosen based on data availability, while simultaneously minimizing the risk of cross-task learning. For instance, all data sets were used for the slide origin prediction task (task 3) except data set 2, as the Department of Dermatology, University Hospital Kiel, used a different scanner type (3DHISTECH scanner; 3DHISTECH Ltd) to digitize the slides (Table 1). Therefore, a classifier could potentially determine the slide origin for data set 2 by determining the scanner type.

\section{Task 1: Patient Age}

To determine patient age, data set 2 was used, and only slides with an assigned patient age were analyzed. Slides were divided into one of two classes based on patient age $(\leq 48$ years versus $>78$ years), excluding slides of patients with ages in between. The cutoff points were chosen in an effort to achieve a natural balance between both age groups, with the 30-year gap making it plausible to observe possible distinct age-dependent morphological features.

\section{Task 2: Slide Preparation Date}

To determine the year of slide preparation, data sets 1 and 2 were used. Data availability varied but was generally sufficient for years 2014-2018. For each data set, separate binary classification tasks were defined, where slides were taken from 
every other year to ensure that there was a minimum of 365 days between the preparation dates of slides from each class (eg, data set 1, 2015 versus 2017). This resulted in five separate classification subtasks.

\section{Task 3: Slide Origin}

To determine the origin of the respective slide, all data sets except data set 2 were used. Origin was defined as the institution from which the slides were obtained.

\section{Task 4: Scanner Type}

To determine the scanner type, data set 4 was used. These slides were scanned twice, but due to the slight difference in resolution between the Zeiss (Carl Zeiss AG) and Hamamatsu (Hamamatsu Photonics KK) scanners, scanned Zeiss slides were reprocessed specifically for this task by downscaling their resolution $(0.22$ $\mu \mathrm{m} / \mathrm{px})$ to match the resolution of the Hamamatsu scanner $(0.23$ $\mu \mathrm{m} / \mathrm{px})$.

\section{Model Training}

Each task had a designated combined data set based on the setup outlined above. Each combined data set was divided into a training set and a test set on slide level using an 80:20 split. If the resulting test set contained fewer than 10 slides, a cross-validation approach on slide level was employed to increase the size of the test set to a minimum of 10 slides per class.

A ResNet50 architecture was trained for each task. In cases where cross-validation was used for testing, the number of trained CNNs equaled the number of cross-validation folds. The training run for each task was repeated a total of five times to obtain a robust average performance uninfluenced by stochastic training events. This number was chosen arbitrarily but with the intention to reduce the overall computing time. For exact technical details on the cross-validation and training procedure, please see Multimedia Appendix 1 or refer to Multimedia Appendix 2 for an exemplary jupyter notebook demonstrating the basic training procedure.

\section{Model Inference and Statistical Evaluation}

Inference was carried out on each task's respective test set using the complete set of tiles for each slide. The class for a WSI was computed by first predicting on its complete set of tiles, averaging all output probabilities, and assigning the class label with the highest average probability to the slide. Because each training and evaluation run for a task was repeated five times, a mean balanced accuracy with a corresponding $95 \%$ confidence interval could be computed.

\section{Results}

Learnability was investigated on the slide level, as that is the standard and decisive criterion in DP. For all tasks, balanced accuracy was generally higher on slide level than on tile level.

For each task, a balanced accuracy over $50.0 \%$ was achieved, even when taking into account the range of the corresponding confidence intervals. Classifier performance varied widely inter-task and intra-task for task 2, which had multiple subtasks. Task 1 (patient age) had a mean balanced accuracy of $87.5 \%$ (Table 2). For task 2 (slide preparation date), performance varied widely between subtasks, ranging from $56.1 \%$ (95\% CI $52.7 \%$ to $59.5 \%$ ) to $83.5 \%$ (95\% CI $80.9 \%$ to $86.1 \%$ ). Classifiers for task 3 (slide origin) and 4 (scanner type) showed balanced accuracies of $97.9 \%$ (95\% CI $97.3 \%$ to $98.5 \%$ ) and $100 \%$, respectively.

Table 2. Overall mean performance of each task's classifiers measured using mean balanced accuracy and evaluated on tile level and slide level.

\begin{tabular}{|c|c|c|}
\hline \multirow[b]{2}{*}{ Task $^{\mathrm{a}}$} & \multicolumn{2}{|c|}{ ResNet50 performance (mean balanced accuracy) } \\
\hline & Tile level & Slide level $(95 \% \mathrm{CI})^{\mathrm{b}}$ \\
\hline 1: Patient age & $76.2 \%$ & $87.5 \%$ \\
\hline \multicolumn{3}{|l|}{ 2: Slide preparation date } \\
\hline Data set 1: 2015 versus 2017 & $54.1 \%$ & $56.1 \%(52.7 \%$ to $59.5 \%)$ \\
\hline Data set 1: 2016 versus 2018 & $56.5 \%$ & $63.2 \%(53.4 \%$ to $73.0 \%)$ \\
\hline Data set 2: 2014 versus 2016 & $69.0 \%$ & $82.0 \%(76.4 \%$ to $87.6 \%)$ \\
\hline Data set 2: 2015 versus 2017 & $66.6 \%$ & $83.5 \%(80.9 \%$ to $86.1 \%)$ \\
\hline Data set 2: 2016 versus 2018 & $52.7 \%$ & $56.7 \%(52.6 \%$ to $60.7 \%)$ \\
\hline 3: Slide origin & $94.2 \%$ & $97.9 \%(97.3 \%$ to $98.5 \%)$ \\
\hline 4: Scanner type & $100 \%$ & $100 \%$ \\
\hline
\end{tabular}

${ }^{\mathrm{a}}$ Test sets for each task had a minimum of 10 slides per class.

${ }^{\mathrm{b}}$ Confidence intervals are shown for the decisive criteria (slide level) and are omitted for tasks where no variation on slide level was observed.

Looking at the distributions of each task-specific model for the first run, slide origin and scanner type could be predicted with very high accuracy, with minor misclassification errors for task 3 (slide origin) and no misclassifications for task 4 (scanner type). For task 1, patient age below 48 years could be predicted with high accuracy, but one-quarter of the slides originating from older patients (>78 years) were erroneously classified as belonging to the younger age group. For task 2 (slide preparation date), the results varied widely between comparisons. The 2-year comparison with the highest balanced accuracy (data set 2, 2015 
versus 2017) showed some misclassifications, with slides from 2015 occasionally being classified as 2017 , whereas the task with the lowest balanced accuracy (data set 1, 2015 versus 2017) showed frequent misclassifications in both directions (Figure 2).

Figure 2. Distributions of the models' predictions for tasks 1 to 4 on slide level for ResNet50 (run 1). (A) Task 1 (patient age) prediction. (B) Task 4 (scanner type) prediction. (C, D) Comparison of the two most distinct classification subtasks for task 2 (slide preparation date), where balanced accuracy was either at a maximum (C: data set 2, 2015 versus 2017) or at a minimum (D: data set 1, 2015 versus 2017). (E) Task 3 (slide origin) prediction, with the data set number displayed rather than the data set origin for better readability.

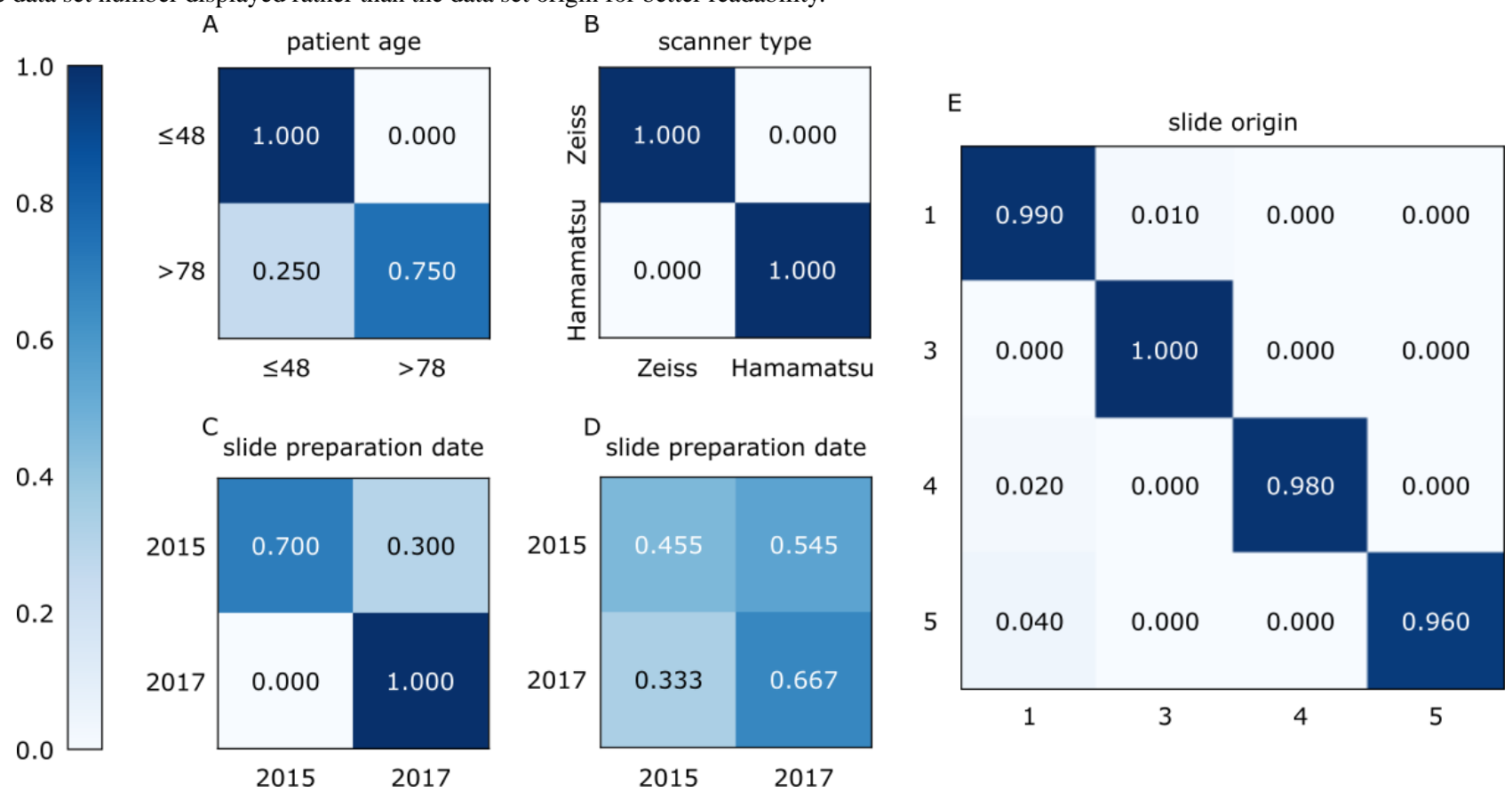

\section{Discussion}

\section{Principle Findings}

Using four exemplary hidden variables found in DP WSI data sets, we showed that these variables were learned by a DL algorithm for a dermatopathological data set. A learnable hidden variable may cause a batch effect, which can greatly affect the training of such algorithms if said variable is unintendedly picked up instead of or in addition to the intended target variable. We hypothesized that there would be hidden variables that would figure very prominently on the slides. These are more likely to be picked up and used by the algorithm to classify the slides and therefore pose the greatest threat to classification accuracy. To identify such "high risk" variables, we limited the amount of training the CNN received, using a standard architecture and a training procedure with little optimization and no training until convergence. This should result in only prominent variables being learned, which likely pose the greatest threat to a classifier's accuracy, although an influence of factors that may be learned by a more extensive training procedure cannot be excluded.

All of the four variables tested (patient age, slide preparation date, slide origin, and scanner type) were learned by the classifier, albeit to different extents. The highest balanced accuracy was observed for task 4 (scanner type). For this task, slides from data set 4 were scanned twice with different devices but processed with the same image-processing pipeline, leaving differences in scanner type (eg, specific scanner hardware or software) as the only causative source of variations, which must be quite pronounced based on the high balanced accuracy.

Performances for tasks 3 (slide origin) and 4 (scanner type) were comparable. As all slides for task 3 were scanned and processed using the same scanner type and pipeline, digitization as the source of the observed batch effects can be ruled out. Therefore, the source of variation most likely stemmed from the slide preparation step, a complex process with lots of potential variables related to the sectioning, fixation, staining, and mounting procedures. Determining the origin of images was previously shown outside the field of DP, where a CNN correctly identified hospital systems based solely on chest radiographs [21].

The aforementioned slide preparation step was also a likely contributor to the "classifiability" of the slide preparation dates (task 2), together with slide aging itself (ie, small amounts of tissue and dye degradation). While slide aging is presumably a gradual process, the slide preparation step is expected to change more abruptly when institutions change the exact mode of slide preparation over time (eg, when a new staining protocol is introduced). This could explain the large variability in classifier performance for task 2, where in some cases the difference between two particular years could be identified much more accurately than between two other years at the same institution. Based on these results, the learnability of this task depends highly on the chosen year and data set, making the underlying variable less of a risk factor but still worthwhile considering.

The patient age prediction task was the only task that reflected a true "biological" difference between the analyzed groups. It 
is known that the texture of the skin changes as result of the aging process via a multitude of processes [22]. For instance, the amount of elastin and collagen decreases with time, which results in a restructuring of the fibrous tissue in deeper skin layers. On UV-exposed skin, additional similar effects may also be induced by photoaging. Moreover, some extent of biological variability regarding the aging process likely exists. This rather complex pattern of skin aging may explain why the ability of the CNN to separate the chosen age groups was not perfect. Performance would likely drop if the age gap of 30 years were to be reduced or if instead of WSIs showing both tumor and healthy skin, only tumor regions were regarded. Nonetheless, the classifier's ability to pick up variables representing large age differences is important to consider, as an unequal patient age distribution is not unlikely to occur for certain medical DL objectives, especially since cancer incidence increases with age.

Based on these findings, it is not unlikely that the discussed variables may interfere with the generation of an accurate CNN-based classifier. Due to the technical nature of slide preparation date, slide origin, and scanner type, their learnability could generalize to other fields in DP, which may have to be investigated in further studies. The learnability of patient age, however, may be more specific to the field of dermatology. While patient age is known to have an impact on the skin, age-related differences may be much less prominent in other tissues.

\section{Prevention and Verification}

In order to minimize the learning of batch effect variables, we suggest to balance any known batch effect variables during creation of the training data set, in addition to any normalization and preprocessing standardization. If easy-to-learn variables are equally balanced between classes, separation based on these variables should no longer result in a reduction of the training loss, thus losing its optimization value. In addition, large and diversified validation sets decrease the likelihood that unwanted correlations between batch effect variables and the intended biological variable exist and thereby can aid in uncovering whether the intended biological variable or some confounding hidden variable was learned.

Unfortunately, balancing training data sets for all potential batch effects is unfeasible. Even randomized clinical trials can only ever be balanced for a few features that are deemed crucial to the comparison in question. With time, additional knowledge accumulating both within and outside the field of AI-supported medicine may help researchers to clarify which potential influencing variables have to be taken into account for which tasks. In this regard, the future realization of more transparent AI-systems that facilitate both explainability and causability [23] would go a long way in helping practitioners to better assess the reliability of AI-systems through a better understanding of their decision-making process.

\section{Limitations}

A major limitation is that the list of considered artifacts is not exhaustive. As numerous other potential confounders exist, some of which we are not yet aware of, complete coverage of all possible artifacts in one study is impossible and therefore has to be limited to a selection considered to be crucial. However, because of the black-box nature of DL algorithms, there is no proof to show what the model actually learned, meaning that any of the unaccounted for artifacts could by chance correlate with a task's class distribution and be learned instead. This cannot be ruled out; however, by increasing validation set size and diversity, chances of these accidental training set correlations persisting through to the validation set decrease and should therefore be detected during the validation stage.

In this study, only results obtained with one DL architecture are shown. We therefore investigated two additional architectures (DenseNet121 and VGG16) and observed a similar trend (see Multimedia Appendix 1).

While learnability was only investigated on a dermatopathological data set in this study, some of the insights gained here may be transferable to other fields in DP. Moreover, this study did not intend to show exactly what variables can be learned but rather to show that unexpected variables can be learned.

\section{Conclusions}

Our DL model was able to learn several potential batch effect variables with relative ease, a finding that is likely to be applicable to other DL models, too. Thus, our results highlight the importance of identifying and minimizing these effects, not only by normalization and preprocessing standardization but also by carefully constructing training and validation sets for DL classification tasks.

\section{Acknowledgments}

This study was funded by the Federal Ministry of Health, Berlin, Germany (grant: Skin Classification Project; grant holder: Dr Titus J Brinker, Digital Biomarkers for Oncology Group, National Center for Tumor Diseases, German Cancer Research Center). The sponsor had no role in the design and conduct of the study; collection, management, analysis, or interpretation of the data; preparation, review, or approval of the manuscript; or decision to submit the manuscript for publication.

\section{Conflicts of Interest}

A Hauschild receives clinical trial support, speaker's honoraria, or consultancy fees from the following companies: Amgen, Bristol Myers Squibb, Merck Serono, MSD, Novartis, Oncosec, Philogen, Pierre Fabre, Provectus, Regeneron, Roche, OncoSec, Sanofi-Genzyme, and Sun Pharma. Outside of the submitted work, JSU is on the advisory board or has received honoraria and travel support from Amgen, Bristol Myers Squibb, GlaxoSmithKline, LeoPharma, Merck Sharp and Dohme, Novartis, Pierre Fabre, and Roche. Outside of the submitted work, SH has advisory roles for or has received honoraria from Pierre Fabre 
Pharmaceuticals, Novartis, Roche, Bristol Myers Squibb, Amgen, and MSD. TJB owns a company that develops mobile apps (Smart Health Heidelberg GmbH, Handschuhsheimer Landstr. 9/1, 69120 Heidelberg).

\section{Multimedia Appendix 1}

Additional methods and results.

[DOCX File, 26 KB-Multimedia Appendix 1]

\section{Multimedia Appendix 2}

Exemplary jupyter notebook.

[ZIP File (Zip Archive), 6 KB-Multimedia Appendix 2]

\section{References}

1. Bera K, Schalper KA, Rimm DL, Velcheti V, Madabhushi A. Artificial intelligence in digital pathology - new tools for diagnosis and precision oncology. Nat Rev Clin Oncol 2019 Nov;16(11):703-715 [FREE Full text] [doi: 10.1038/s41571-019-0252-y] [Medline: 31399699]

2. Ehteshami Bejnordi B, Veta M, Johannes van Diest P, van Ginneken B, Karssemeijer N, Litjens G, the CAMELYON16 Consortium, et al. Diagnostic Assessment of Deep Learning Algorithms for Detection of Lymph Node Metastases in Women With Breast Cancer. JAMA 2017 Dec 12;318(22):2199-2210 [FREE Full text] [doi: 10.1001/jama.2017.14585] [Medline: 29234806]

3. Cruz-Roa A, Gilmore H, Basavanhally A, Feldman M, Ganesan S, Shih NNC, et al. Accurate and reproducible invasive breast cancer detection in whole-slide images: A Deep Learning approach for quantifying tumor extent. Sci Rep 2017 Apr 18;7:46450 [FREE Full text] [doi: 10.1038/srep46450] [Medline: 28418027]

4. Coudray N, Ocampo PS, Sakellaropoulos T, Narula N, Snuderl M, Fenyö D, et al. Classification and mutation prediction from non-small cell lung cancer histopathology images using deep learning. Nat Med 2018 Oct;24(10):1559-1567. [doi: 10.1038/s41591-018-0177-5] [Medline: 30224757]

5. Hekler A, Utikal JS, Enk AH, Berking C, Klode J, Schadendorf D, et al. Pathologist-level classification of histopathological melanoma images with deep neural networks. Eur J Cancer 2019 Jul;115:79-83 [FREE Full text] [doi:

10.1016/j.ejca.2019.04.021] [Medline: $\underline{31129383]}$

6. Hekler A, Utikal JS, Enk AH, Solass W, Schmitt M, Klode J, et al. Deep learning outperformed 11 pathologists in the classification of histopathological melanoma images. Eur J Cancer 2019 Sep;118:91-96 [FREE Full text] [doi: 10.1016/j.ejca.2019.06.012] [Medline: $\underline{31325876]}$

7. Kather JN, Pearson AT, Halama N, Jäger D, Krause J, Loosen SH, et al. Deep learning can predict microsatellite instability directly from histology in gastrointestinal cancer. Nat Med 2019 Jul;25(7):1054-1056. [doi: 10.1038/s41591-019-0462-y] [Medline: $\underline{31160815}$ ]

8. Higgins C. Applications and challenges of digital pathology and whole slide imaging. Biotech Histochem 2015 Jul;90(5):341-347. [doi: 10.3109/10520295.2015.1044566] [Medline: 25978139]

9. Madabhushi A, Lee G. Image analysis and machine learning in digital pathology: Challenges and opportunities. Med Image Anal 2016 Oct;33:170-175 [FREE Full text] [doi: 10.1016/j.media.2016.06.037] [Medline: 27423409]

10. Tizhoosh HR, Pantanowitz L. Artificial Intelligence and Digital Pathology: Challenges and Opportunities. J Pathol Inform 2018;9:38 [FREE Full text] [doi: 10.4103/jpi.jpi 53 18] [Medline: $\underline{30607305]}$

11. Goh WWB, Wang W, Wong L. Why Batch Effects Matter in Omics Data, and How to Avoid Them. Trends Biotechnol 2017 Jun;35(6):498-507. [doi: 10.1016/j.tibtech.2017.02.012] [Medline: 28351613]

12. Phan JH, Quo CF, Cheng C, Wang MD. Multiscale integration of -omic, imaging, and clinical data in biomedical informatics. IEEE Rev Biomed Eng 2012;5:74-87 [FREE Full text] [doi: 10.1109/RBME.2012.2212427] [Medline: 23231990]

13. Taqi SA, Sami SA, Sami LB, Zaki SA. A review of artifacts in histopathology. J Oral Maxillofac Pathol 2018;22(2):279 [FREE Full text] [doi: 10.4103/jomfp.JOMFP 125 15] [Medline: $\underline{30158787]}$

14. Janowczyk A, Basavanhally A, Madabhushi A. Stain Normalization using Sparse AutoEncoders (StaNoSA): Application to digital pathology. Comput Med Imaging Graph 2017 Apr;57:50-61 [FREE Full text] [doi: 10.1016/j.compmedimag.2016.05.003] [Medline: 27373749]

15. Kothari S, Phan JH, Stokes TH, Osunkoya AO, Young AN, Wang MD. Removing batch effects from histopathological images for enhanced cancer diagnosis. IEEE J Biomed Health Inform 2014 May;18(3):765-772 [FREE Full text] [doi: 10.1109/JBHI.2013.2276766] [Medline: 24808220]

16. Kothari S, Phan JH, Wang MD. Scale normalization of histopathological images for batch invariant cancer diagnostic models. Annu Int Conf IEEE Eng Med Biol Soc 2012;2012:4406-4409 [FREE Full text] [doi: 10.1109/EMBC.2012.6346943] [Medline: 23366904]

17. Roy S, Kumar Jain A, Lal S, Kini J. A study about color normalization methods for histopathology images. Micron 2018 Nov;114:42-61. [doi: 10.1016/j.micron.2018.07.005] [Medline: 30096632] 
18. Van Eycke Y, Allard J, Salmon I, Debeir O, Decaestecker C. Image processing in digital pathology: an opportunity to solve inter-batch variability of immunohistochemical staining. Sci Rep 2017 Feb 21;7:42964 [FREE Full text] [doi: 10.1038/srep42964] [Medline: 28220842]

19. Kothari S, Phan J, Moffitt R, Stokes T, Hassberger S, Chaudry Q, et al. Automatic batch-invariant color segmentation of histological cancer images. Proc IEEE Int Symp Biomed Imaging 2011;2011:657-660 [FREE Full text] [doi: 10.1109/ISBI.2011.5872492] [Medline: 27532016]

20. Janowczyk A, Zuo R, Gilmore H, Feldman M, Madabhushi A. HistoQC: An Open-Source Quality Control Tool for Digital Pathology Slides. JCO Clin Cancer Inform 2019 Apr;3:1-7 [ FREE Full text] [doi: 10.1200/CCI.18.00157] [Medline: 30990737]

21. Zech JR, Badgeley MA, Liu M, Costa AB, Titano JJ, Oermann EK. Variable generalization performance of a deep learning model to detect pneumonia in chest radiographs: A cross-sectional study. PLoS Med 2018 Nov;15(11):e1002683 [FREE Full text] [doi: 10.1371/journal.pmed.1002683] [Medline: $\underline{\text { 30399157] }}$

22. Rittié L, Fisher GJ. Natural and sun-induced aging of human skin. Cold Spring Harb Perspect Med 2015 Jan 05;5(1):a015370 [FREE Full text] [doi: 10.1101/cshperspect.a015370] [Medline: 25561721]

23. Holzinger A, Langs G, Denk H, Zatloukal K, Müller H. Causability and explainability of artificial intelligence in medicine. Wiley Interdiscip Rev Data Min Knowl Discov 2019;9(4):e1312 [FREE Full text] [doi: 10.1002/widm.1312] [Medline: 32089788]

\section{Abbreviations \\ AI: artificial intelligence \\ CNN: convolutional neural network \\ DL: deep learning \\ DP: digital pathology \\ ML: machine learning \\ TCGA: The Cancer Genome Atlas \\ WSI: whole slide image}

Edited by G Eysenbach; submitted 12.08.20; peer-reviewed by A Böer-Auer, JA Benítez-Andrades, A Holzinger; comments to author
10.10.20; revised version received 14.10.20; accepted 06.12.20; published 02.02.21
Please cite as:
Schmitt M, Maron RC, Hekler A, Stenzinger A, Hauschild A, Weichenthal M, Tiemann M, Krahl D, Kutzner H, Utikal JS, Haferkamp
S, Kather JN, Klauschen F, Krieghoff-Henning E, Fröhling S, von Kalle C, Brinker TJ
Hidden Variables in Deep Learning Digital Pathology and Their Potential to Cause Batch Effects: Prediction Model Study
J Med Internet Res $2021 ; 23(2):$ e23436
URL: $\underline{\text { https://www.jmir.org/2021/2/e23436 }}$
doi: $\underline{10.2196 / 23436}$
PMID: $\underline{33528370}$

(C) Max Schmitt, Roman Christoph Maron, Achim Hekler, Albrecht Stenzinger, Axel Hauschild, Michael Weichenthal, Markus Tiemann, Dieter Krahl, Heinz Kutzner, Jochen Sven Utikal, Sebastian Haferkamp, Jakob Nikolas Kather, Frederick Klauschen, Eva Krieghoff-Henning, Stefan Fröhling, Christof von Kalle, Titus Josef Brinker. Originally published in the Journal of Medical Internet Research (http://www.jmir.org), 02.02.2021. This is an open-access article distributed under the terms of the Creative Commons Attribution License (https://creativecommons.org/licenses/by/4.0/), which permits unrestricted use, distribution, and reproduction in any medium, provided the original work, first published in the Journal of Medical Internet Research, is properly cited. The complete bibliographic information, a link to the original publication on http://www.jmir.org/, as well as this copyright and license information must be included. 